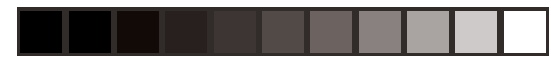

\title{
MAMÍFEROS DEL PARQUE NACIONAL LAGUNAS DE MONTEBELLO, CHIAPAS, MÉXICO
}

\author{
ANNA HORVATH ${ }^{1}$, ROBERTO VIDAL-LOPEZ² Y RAUSEL SARMIENTO- \\ AGUILAR $^{3}$ \\ ${ }^{1}$ División de Conservación de la Biodiversidad, El Colegio de la Frontera Sur (ECOSUR), \\ Carretera Panamericana y Periférico Sur S/N, Apartado Postal 63, CP. 29290, San \\ Cristóbal de Las Casas, Chiapas, MÉXICO. \\ ${ }^{2}$ Dirección actual: Instituto de Historia Natural y Ecología (IHNyE), Calzada de los \\ Hombres Ilustres S/N, C.P. 29000, Tuxtla Gutiérrez, Chiapas, MÉXICO. \\ ${ }^{3}$ Dirección actual: Programa Regional en Manejo de Vida Silvestre para Mesoamérica y el \\ Caribe, Universidad Nacional, Apdo. 1350, Heredia, COSTA RICA.
}

Resumen: En este trabajo damos a conocer la diversidad de mamíferos en el Parque Nacional Lagunas de Montebello, Chiapas, con información sobre su distribución local por tipos de vegetación, afinidad de distribución geográfica y estado de conservación. Los datos se obtuvieron a través de capturas y observaciones en el campo, completándose con información bibliográfica y de bases de datos. Se registraron 52 especies de mamíferos silvestres, comprendidos en 7 órdenes, 18 familias y 41 géneros, los òrdenes mejor representados son Rodentia, Chiroptera y Carnivora. Las familias más diversas son Muridae y Phyllostomyidae, y los géneros más diversos son Peromyscus y Reithrodontomys. Esta riqueza constituye el 11.2\% del total de los mamíferos terrestres de México y el 25.2\% de los mamíferos de Chiapas. El 58\% de las especies son nuevos registros para el Parque. La mastofauna de Montebello muestra una afinidad Neotropical con una buena representación de especies mesoamericanas, incluyendo dos especies endémicas de México. La comunidad más diversa se encuentra en el bosque mesófilo de montaña, mostrando un alto nivel de asociación entre las especies y el tipo de vegetación. Los mamíferos de Montebello están poco representados en los diversos listados de estatus de conservación, ya que algunas de las especies que aparecen en ellos ya están localmente extintas del área.

Abstract: In this paper we summarize the information available on diversity of mammals in the Lagunas de Montebello National Park, Chiapas, Mexico, including data about the local distribution by vegetation types, geographical affinity by their distribution ranges and conservation status. Data were obtained by captures and observations in the field, and were completed with bibliographical as well as data-base information. 52 species of native mammals were recorded, arranged in seven orders, 18 families and 41 genera. This species richness constitutes the $11.2 \%$ of terrestrial mammals of Mexico and the $25.2 \%$ of the mammal diversity of Chiapas. The $58 \%$ of the species are new records for the Park and the orders with more 
representation are Rodentia, Chiroptera and Carnivora. The more diverse families are Muridae and Phillostomyidae while the most species genera were Peromyscus and Reithrodontomys. The fauna of mammals of Montebello shows a Neotropical affinity and represents a high portion of Mesoamerican mammals including two Mexican endemic species. The most diverse community was encountered in the tropical mountain rainforest, showing a high association between species and the vegetation types. The mammals of Montebello have a relatively little representation in the different lists of conservational status due to the recently local extinctions of species from the area.

Palabras clave: mastofauna, diversidad, distribución, estado de conservación, Parque Nacional Lagunas de Montebello, Chiapas, México.

\section{INTRODUCCIÓN}

Las Áreas Naturales Protegidas (ANP) fueron creadas con el objeto de preservar los ambientes naturales representativos de las diferentes regiones biogeográficas, ecológicas y ecosistemas de mayor fragilidad en México. El objetivo principal de una ANP en términos generales es asegurar la continuidad de los procesos ecológicos y la conservación y aprovechamiento sostenible de la biodiversidad (Peña-Jiménez et al., 1998). Sin embargo, la mayoría de las ANPs en México enfrentan una serie de problemas que afectan su conservación y manejo adecuado. Dentro de la problemática compleja de las ANPs se suman los conflictos de la tenencia de la tierra, la carencia de vigilancia y planes de manejo, así como la escasa información sobre los recursos bióticos que albergan y las condiciones en que estas se encuentran en la actualidad. En este sentido, el estudio integral de las ANPs es de prioridad para el conocimiento y conservación de las especies y los sistemas ecológicos a los que pertenecen; y de este modo contar con información necesaria para proponer alternativas para el aprovechamiento racional y conservación a largo plazo de la riqueza biológica (Cervantes et al., 1995).

El Parque Nacional Lagunas de Montebello (PNLM) es una de las primeros ANPs en México, ya que se decretó en 1959. Sorprendentemente y a pesar de su historia de casi medio siglo, se conocen pocos estudios faunísticos y ecológicos sobre este parque (Ruíz et al., 1998). El conocimiento que se tiene sobre los mamíferos en Montebello, hasta la fecha lo constituyen estudios recientes sobre algunos aspectos particulares de ecología poblacional y de comunidades de roedores (Horváth y Navarrete, 1997; Horváth et al., 2001; Reyes, 2001; Sarmiento, 1999). Por lo tanto, este trabajo tiene la finalidad de dar a conocer la diversidad de mamíferos silvestres del Parque, enfatizando sobre la distribución local por tipo de vegetación, el estado de conservación y la importancia de las especies. 
vol. 5

\section{MÉTODOS}

\section{Área de estudio}

El PNLM fue decretado como tal el 16 de diciembre de 1959 (Secretaria de Agricultura y Ganadería, 1959). Se ubica en la parte Este-Sureste del Estado de Chiapas, a los $16^{\circ} 10^{\prime} 20^{\prime \prime}$ y $16^{\circ} 04^{\prime} 40^{\prime \prime}$ de latitud Norte y a los $91^{\circ} 47^{\prime} 40^{\prime \prime}$ y $91^{\circ} 37^{\prime} 40^{\prime \prime}$ de longitud Oeste; limitando al Sur con Guatemala. Abarca una superficie de 6,022 ha, dentro de los municipios de La Trinitaria y La Independencia (Figura 1), teniendo una altitud promedio de 1,500 msnm. La vegetación dominante corresponde a bosques de climas templado-húmedo, predominando las asociaciones de Pino y Pino-Encino-

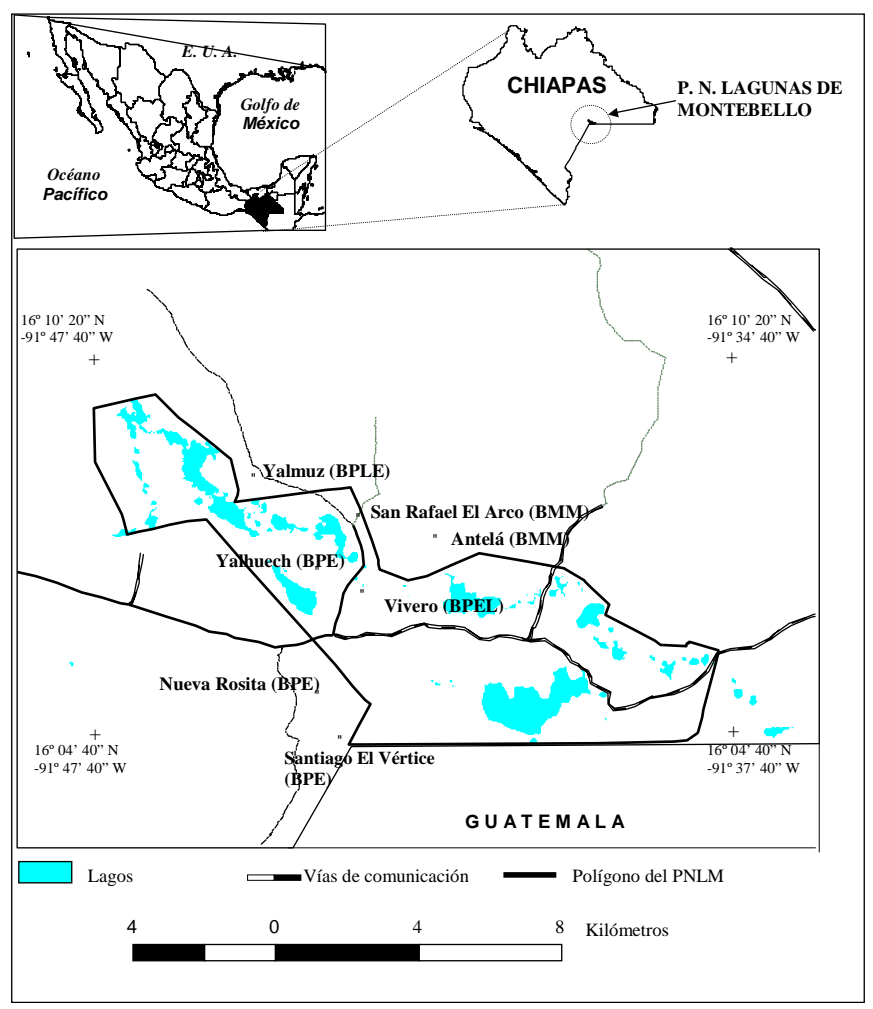

Figura 1. Localización del Parque Nacional Lagunas de Montebello en Chiapas, México. 
Liquidámbar; también se encuentra el Bosque Mesófilo, con elementos perennifolios, como los encinos, y caducifolios como el liquidámbar (Carlson, 1954).

\section{Muestreo de mamíferos}

Durante el período de febrero de 1997 a marzo de 2000 se realizaron 32 visitas de trabajo de campo al PNLM. Los muestreos comprendieron siete sitios los cuales se eligieron por la presencia de los tres tipos de vegetación naturales de mayor representatividad: Bosque de Pino-Encino (BPE), Bosque de Pino-EncinoLiquidámbar (BPEL) y Bosque Mesófilo de Montaña (BMM). También se tomó en cuenta el acceso y consentimiento de las comunidades locales para el trabajo de campo (Figura 1). Las visitas incluyeron proporcionalmente las épocas de sequía y de lluvias. Además se compiló información sobre el conocimiento previo de la mastofauna del Parque, basado en registros bibliográficos y bases de datos de las colecciones mastozoológicas de El Colegio de la Frontera Sur (ECOSUR: ECO-SCM) y del Instituto de Historia Natural y Ecología del Estado (IHNyE).

Los mamíferos pequeños no voladores se capturaron con trampas tipo Sherman para ejemplares vivos. Las trampas se cebaron con avena mezclada con crema de cacahuate y esencia de vainilla. Los muestreos se llevaron a cabo en cuadrantes, uno por sitio de muestreo. En cada cuadrante se estableció una retícula de trampeo que contenía 100 trampas, con diez líneas separadas cada diez metros, de diez trampas por línea. En cada sitio se llevaron a cabo los muestreos mensuales con los siguientes intervalos: de febrero a octubre de 1996 en nueve sitios se efectuaron tres repeticiones con tres días de captura-recaptura (esfuerzo de captura $=4,050$ noches-trampa); de junio de 1997 a marzo de 1998 en cuatro sitios se efectuaron seis repeticiones con tres días de captura-recaptura (esfuerzo de captura $=7,200$ noches-trampa); de abril a julio de 1999 en un sitio se repitió cuatro veces con seis días de captura-recaptura (esfuerzo de captura $=2,400$ noches-trampa). Las trampas se revisaron dos veces al día, aproximadamente a las 07:00 hrs. y a las 17:00 hrs. El esfuerzo de captura total para toda el área fue de 13,650 noches-trampa.

Para la captura de mamíferos medianos, se colocaron transectos de 20 trampas Tomahawk cebadas con frutas (guayaba, mango y plátano) y sardina, alrededor de los cuadrantes de trampeo para roedores. Los murciélagos fueron capturados con redes de 6 y de $12 \mathrm{~m}$, colocadas dentro de las parcelas y en algunos lugares estratégicos cerca de ellas (entrada de grutas, orillas de cuerpos de agua o veredas), en los siguientes intervalos de muestreo mensual: de marzo de 1997 a septiembre de 1998 en cuatro sitios se colocaron tres redes durante cinco horas por tres noches (esfuerzo de captura $=2,160$ metros de red por hora); en abril de 1999 en un sitio se colocaron 9 redes (una noche) y 6 redes (una noche) durante cinco horas (esfuerzo de captura $=$ 730 metros de red por hora); en noviembre de 1999 en un sitio se colocaron ocho 
redes durante cinco horas (esfuerzo de captura $=840$ metros de red por hora) y en marzo del 2000 en un sitio se colocaron cinco redes durante siete horas (esfuerzo de captura $=308$ metros de red por hora). El esfuerzo de captura total para toda el área fue de 4,110 metros de red por hora. Asimismo, se obtuvieron registros de mamíferos por medio de recorridos nocturnos, camas de arena cebadas con huevo fermentado colocadas en transectos permanentes, y animales muertos o sus restos que fueron encontrados.

La determinación taxonómica de los individuos capturados se llevó a cabo basándose en las características externas y con apoyo de las claves de Hall (1981) y Huckaby (1980) y las guías de campo de Aranda y March (1987), Medellín et al. (1997) y Reid (1997). Para cada individuo capturado se tomaron las medidas somáticas usuales para mamíferos, la localidad, fecha, hora, especie, número individual, número de trampa o número de red, sexo, clase de edad y estado reproductivo. A los individuos capturados se aplicó marcaje individual permanente con muescas en las orejas para los roedores y marsupiales (Horváth et al., 2001) y marcaje semipermanente con collares en murciélagos (M. Amín y R. Medellín, in litt.). Después de la toma de datos y marcaje, los individuos fueron liberados en el lugar de captura.

Únicamente se realizaron las colectas mínimas necesarias para la identificación segura a nivel de especie de algunos roedores y murciélagos, así también se colectaron los restos de animales encontrados muertos. Los ejemplares colectados se depositaron en la Colección Mastozoológica de ECOSUR.

\section{Análisis}

Para el almacenamiento y facilitación del análisis de la información se elaboró una base de datos con el programa ACCESS ${ }^{\circledR}$. Se consideraron los siguientes tipos de registros: nuevo registro para el PNLM; registrado por la Colección Mastozoológica de IHNyE y que no fue encontrado durante este estudio; registrado por la Colección Mastozoológica de IHNyE y también durante el periodo de estudio; registrado por Horváth y Navarrete (1997) y registrado por Horváth et al. (2001). La distribución de las especies se clasificó en cinco categorías: compartidas con Norteamérica, compartidas con Sudamérica, compartidas con Norte y Sudamérica, endémicas de México y endémicas de Mesoamérica (Arita y Ceballos, 1997; Flores-Villela y Gerez, 1988). El estado de conservación a nivel nacional se basó en la Norma Oficial Mexicana NOM-059-ECOL-2001 (SEMARNAT, en prensa) donde están consideradas las categorías: en peligro de extinción, amenazada y sujeta a protección especial. En tanto que para el estado de conservación en el ámbito mundial, la información se basó en la Convención Internacional sobre Tráfico de Especies en Peligro de Flora y Fauna Silvestres (CITES, 1995) y la Unión Internacional para la 
Conservación de la Naturaleza (UICN; Baillie y Groombridge, 1996). Las categorías consideradas en este trabajo para CITES son: especies con problemas de conservación, que son o podrían ser afectadas por el tráfico internacional (Apéndice I), especies que podrían verse amenazadas con la extinción si no se controla su tráfico (Apéndice II) y especies reguladas por alguno de los socios del tratado (Apéndice III). La clasificación de la UICN considera a las especies en las categorías de extinta, extinta en estado silvestre, críticamente amenazada, en peligro, vulnerable y en menor riesgo o casi amenazada.

Para la estimación y comparación de las abundancias relativas se utilizaron índices de abundancia relativa (IAR) en caso de didélphidos, roedores y quirópteros, mientras que para los demás mamíferos se utilizó el número de individuos o rastros observados. Para calcular el IAR para los pequeños mamíferos no voladores se dividió el número total de individuos capturados entre el esfuerzo total de capturas (noches-trampa), multiplicados por 100; y en los murciélagos las abundancias se obtuvieron al dividir el número de individuos capturados entre los metros de red por hora. La información de las abundancias relativas comprenden para todo el Parque.

\section{RESULTADOS}

En el PNLM se han registrado 52 especies de mamíferos silvestres, comprendidos en siete órdenes (70\% del total nacional), 18 familias (49\%) y 41 géneros (25.3 \%). Estas especies constituyen el $11.2 \%$ del total de las especies terrestres de México y el 25.2\% de los mamíferos que habitan en Chiapas (Cuadro 1, Figura 2). De las especies registradas en este trabajo, más de la mitad son nuevos registros para el Parque (30 especies, 58\%); de éstas, dos son didélfidos, un insectívoro, nueve quirópteros, nueve carnívoros, un artiodáctilo, siete roedores y un lagomorfo (Apéndice). Los órdenes mejor representados son los roedores (20 especies, 38\% de los mamíferos del PNLM), murciélagos (17 especies, 33\%) y carnívoros (9 especies, 17\%). Las familias más diversas son la Muridae y Phyllostomyidae con 14 especies cada una; mientras que los géneros más diversos son Peromyscus y Reithrodonthomys (Rodentia: Muridae) con cuatro y tres especies, respectivamente, que en su conjunto equivalen al 13\% de los mamíferos de Montebello.

Se encontró que en el PNLM habitan dos especies de mamíferos endémicas de México (Peromyscus zarhynchus y P. melanophrys), de las cuales $P$. zarhynchus tiene distribución restringida en Chiapas. Además el 27\% de las especies son endémicas de Mesoamérica (un marsupial, un carnívoro, dos murciélagos y 10 roedores), 20 especies muestran distribución compartida únicamente con Sudamérica (38\%), 15 especies con Norte y Sudamérica (28\%) y solo una se comparte con Norteamérica (2\%; Apéndice). 
vol. 5

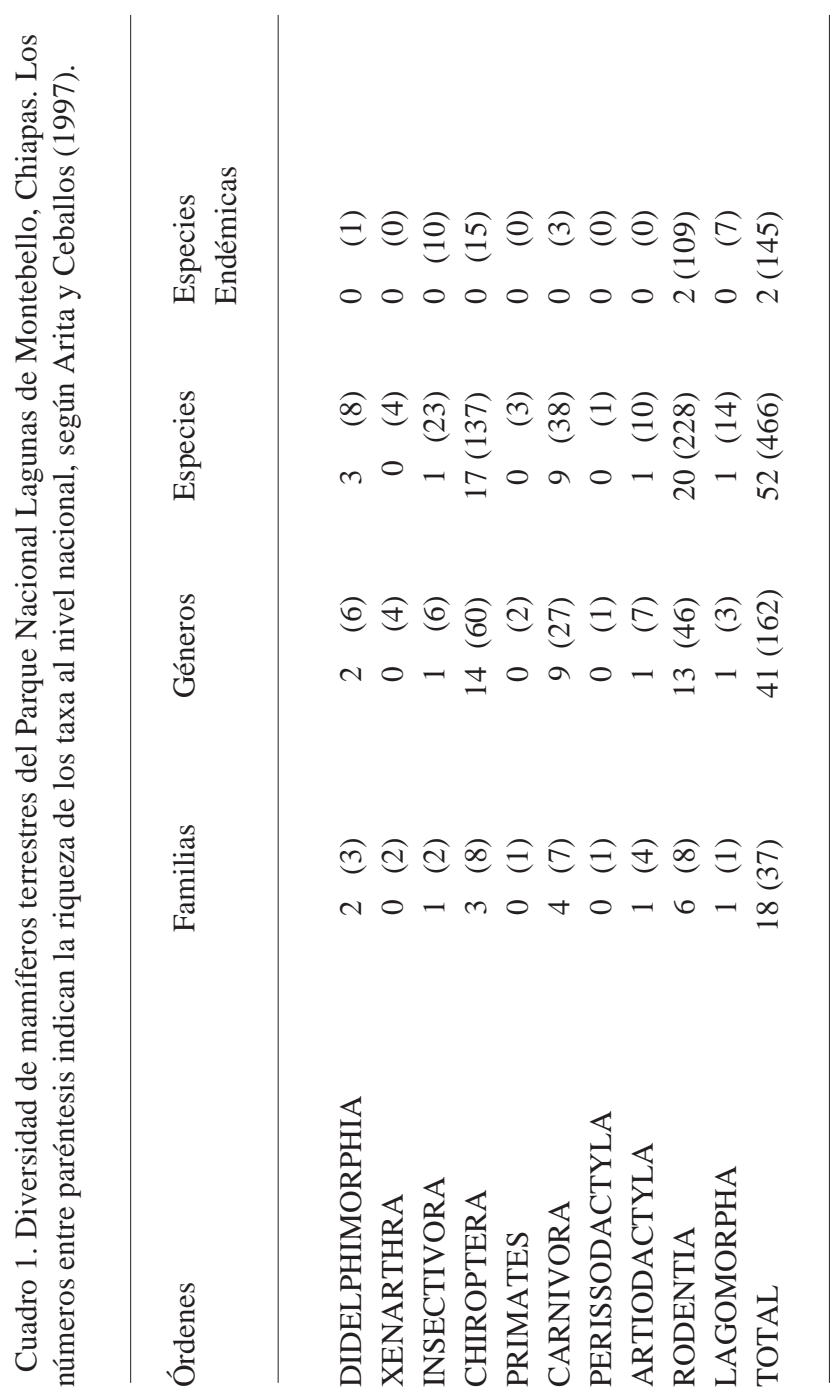




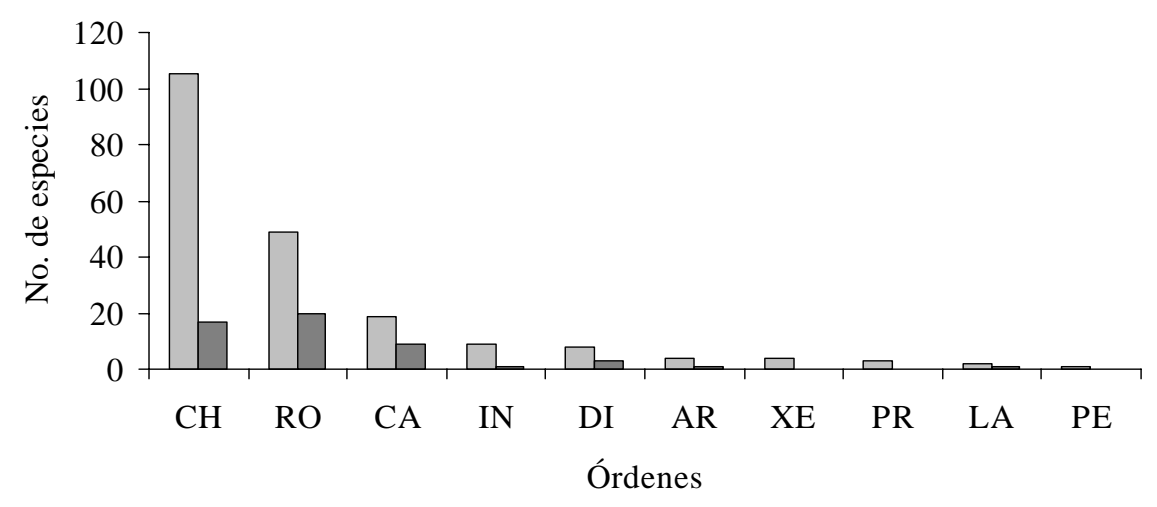

$\square$ Chiapas $\square$ Lagos de Motebello

Figura 2. Número de especies por órdenes en el Parque Nacional Lagos de Montebello y en Chiapas. $\mathrm{CH}=$ Chiroptera, $\mathrm{RO}=$ Rodentia, $\mathrm{CA}=$ Carnivora, $\mathrm{IN}=$ Insectivora, $\mathrm{DI}=$ Didelphimorphia, $\mathrm{AR}=$ Artiodactyla, $\mathrm{XE}=$ Xenarthra, $\mathrm{PR}=$ Primates, $\mathrm{LA}=$ Lagomorpha, $\mathrm{PR}$ $=$ Perissodactyla.

En la riqueza por tipos de vegetación, la fauna más diversa se encontró en el BMM, con el 77\% de la mastofauna del PNLM (40 especies). Las dos asociaciones vegetales restantes, BPEL (29 especies) y BPE (20 especies) albergaron el 57\% y 38\% de los mamíferos, respectivamente. Aunque la riqueza de los tres tipos de vegetación es diferente, cuatro grupos predominan en la composición de sus faunas (Figura 3). Los roedores constituyen el 55\% en el BPE, 52\% en BPEL y 42\% en BMM; los murciélagos conforman el 35\% de las especies del BMM y el 21\% en BPEL; los carnívoros comprenden el 25\% de las especies en el BPE y el 10\% en BPEL y BMM; y por último, los marsupiales integran el 10\% de las especies en el BPE y BPEL, y el 7\% en el BMM.

Más de la mitad de las especies de mamíferos de Montebello se encontró únicamente en un tipo de vegetación (27 spp exclusivas, 52\%) y menos de la cuarta parte (12 spp, 23\%) ocupó los tres tipos de vegetación. De las especies exclusivas a un tipo de vegetación, la mayoría fue para el BMM con 16 especies, que representa el $59 \%$ de las especies exclusivas y $30 \%$ de la riqueza total. Los BPEL y BPE presentaron menos exclusividad con 6 y 5 especies respectivamente que representó el $11 \%$ de los mamíferos de Montebello.

La mayoría de los registros de mamíferos medianos y grandes fueron observaciones directas o indirectas así como animales encontrados muertos y se registraron con frecuencias bajas. 


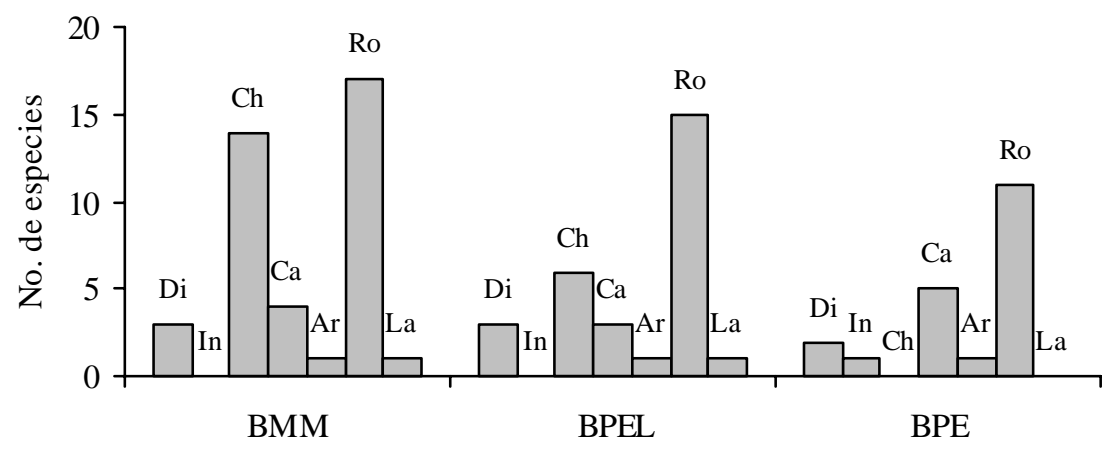

Tipos de Vegetación

Figura 3. Número de especies por orden en los diferentes tipos de vegetación. $\mathrm{CH}=$ Chiroptera, RO = Rodentia, CA = Carnivora, IN = Insectivora, DI = Didelphimorphia, AR = Artiodactyla, $\mathrm{XE}=$ Xenarthra, $\mathrm{PR}=$ Primates, $\mathrm{LA}=$ Lagomorpha, $\mathrm{PR}=$ Perissodactyla .

En algunos casos la presencia de la especie se registró en base a solo un avistamiento o un ejemplar encontrado muerto durante el estudio. Es el caso de los félidos (Herpailurus yagouaroundi y Leopardus wiedii), el puercoespín (Coendou mexicanus), el cacomixtle (Bassariscus sumichrasti) y el coatí (Nasua narica). Por lo anterior, para el análisis de las abundancias relativas únicamente se consideraron los grupos de los cuales se obtuvo mayor número de datos a través de capturas: los marsupiales, roedores múridos y quirópteros. El análisis de dicha información mostró que entre los mamíferos no voladores Reithrodontomys mexicanus, Peromyscus zarhynchus y Oryzomys alfaroi tuvieron la mayor abundancia en toda el área (Cuadro 2 ). En el caso de $P$. zarhynchus cabe mencionar que su alta abundancia en general se debió a que es la especie localmente más abundante, pero sólo se encontró en los bosques mesófilo y de pino-encino-liquidámbar bien conservados (Cuadro 2, Apéndice). Los marsupiales por su parte fueron poco abundantes, con excepción de Didelphis virginiana, la especie mejor representada de este grupo (Cuadro 2). Los murciélagos en general fueron poco abundantes, encontrándose como especies dominantes a Anoura geoffroyi, Sturnira ludovici, Diphylla ecaudata y Desmodus rotundus, que representaron el 72\% del total de murciélagos capturados (Cuadro 3). Las dos últimas especies ( $D$. ecaudata y $D$. rotundus) son importantes en sanidad animal. 

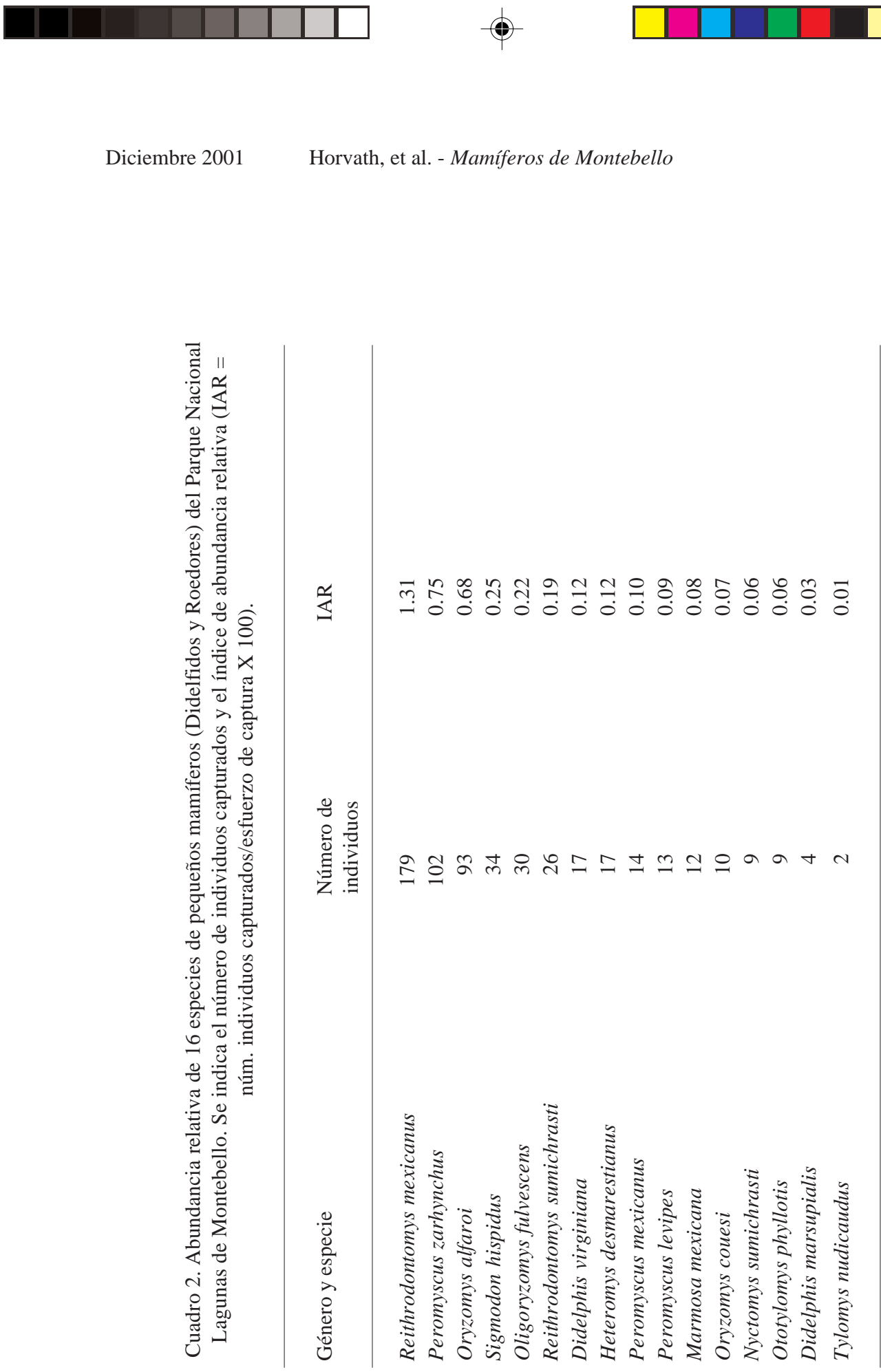
Únicamente 11 especies se encuentran asignadas a una categoría de protección, que corresponde al 21\% de la mastofauna de PNLM. En el ámbito nacional seis están consideradas por la NOM-059-ECOL-2001 (SEMARNAT, en prensa), mientras que ocho especies están incluidas en las listas del CITES y tres en la del IUNC. Los mamíferos bajo protección corresponden a carnívoros (5 especies), roedores (3 especies) y murciélagos (2 especies); de estas, cuatro especies están consideradas en dos o tres instancias reguladoras, tres especies solo por el CITES y dos especies solo por la SEMARNAT (Apéndice).

\section{DISCUSIÓN}

La riqueza de la mastofauna en Montebello es menor comparada con otras ANPs de alta biodiversidad en Chiapas, como Montes Azules, El Triunfo y El Ocote. En esas reservas de la biosfera la riqueza de mamíferos es el doble de la que se registró en Montebello (Espinoza et al., 1998; Medellín, 1994; Navarrete et al., 1996; Zarza, 2001). Sin embargo y considerando el tamaño del PNLM, que es aproximadamente $10 \%$ de El Ocote, $5 \%$ de El Triunfo y 2\% de Montes Azules, y que está inmerso en un paisaje altamente fragmentado, hace evidente la importancia de este Parque. El PNLM, por su ubicación geográfica en la zona de transición entre las regiones de la Meseta Central de Chiapas y la Selva Lacandona, así como por las condiciones fisicoambientales que lo caracteriza y como resultado de esto los tipos de hábitat naturales que se encuentran en su área, constituye un importante refugio para la biodiversidad del sureste mexicano. Esta importancia no solo es notable por el número de especies que potencialmente se podrían encontrar en la zona por su distribución geográfica, sino también porque presenta una mezcla de especies de los bosques templados y de las selvas tropicales perennifolias, concentrada en una superficie relativamente pequeña, encontrándose en los límites más altos o más bajos de su distribución altitudinal. De esta forma, se favorece que las comunidades ecológicas que están presentes en la zona de Montebello, tengan una composición particular y característica para esta área, diferente tanto a las comunidades de las selvas tropicales como las que se encuentran en las zonas de los bosques templados (Carlson, 1954; Horváth et al., 2001). Así, por ejemplo, se encuentra a 1460 msnm, el registro más bajo conocido de Peromyscus zarhynchus coexistiendo con Nyctomys sumichrasti, Tylomys nudicaudus y Oryzomys couesi, composición que no se ha reportado en otros lugares (Baker et al., 1971; Smith y Jones, 1967; Vidal, 1998).

La mastofauna del área muestra claramente una tendencia de afinidad neotropical y alberga una buena representación de los mamíferos mesoamericanos. El alto porcentaje de especies endémicas mesoamericanas y la presencia de las dos especies endémicas a México subraya la importancia de la zona de Montebello, ya que en general, Chiapas no está considerado como una región con alto nivel de endemismos 
de mamíferos a nivel nacional; sin embargo tiene una destacada importancia en cuanto a endemismos mesoamericanos (March et al., 1995; Retana y Lorenzo, 2001).

Se considera que para los roedores nuestro listado es completo ya que es el grupo sobre el cual se han realizado trabajos intensos en los últimos años (Horváth y Navarrete, 1997; Horváth et al., 2001; Reyes, 2001; Sarmiento, 1999). Cabe mencionar que con las 20 especies registradas, el PNLM alberga una mayor riqueza de roedores de lo que se reporta para Montes Azules (17 especies; Zarza, 2001) y es similar a la riqueza de roedores de El Ocote (21 especies, Navarrete et al., 1996). Para los murciélagos se considera que el número de especies registradas podría aumentar con la utilización de otros métodos de muestreo que en este estudio no se aplicaron (Thomas y LaVal, 1988; Wilson et al., 1996). En caso de los mamíferos medianos y grandes, consideramos que se podrían registrar algunas especies adicionales de acuerdo a información de los pobladores locales. De esta forma probablemente se logre registrar el armadillo (Dasypus novemcinctus), el viejo del monte (Eira barbara), y tal vez la ardilla voladora (Glaucomys volans; E. Espinoza, com. pers.).

Es innegable que actualmente el PNLM y sus alrededores están en una situación crítica ya que sus bosques se encuentran muy fragmentados y alterados tanto por las actividades humanas y los incendios forestales, así como por plagas forestales como el descortezador del pino (Dendroctonus sp.). Por la acelerada pérdida de hábitat naturales aunado con la fuerte presión de la cacería, varias especies de mamíferos que hasta hace unas décadas todavía se habían visto en la zona (el puma, el jaguar, el mono araña y el pecarí de collar; Melo y Cervantes, 1986; R. Castellanos, com. pers.) ya no se encuentran. Otras especies que todavía son aprovechadas directamente por la gente local, son el venado cola blanca (Odocoileus virginianus) y el tepezcuintle (Agouti paca).

La gran riqueza local y alto porcentaje de especies exclusivas de un sólo tipo de vegetación natural sugiere un alto nivel de complementariedad o recambio de especies (diversidad beta) dentro del área de estudio. Es notable la importancia del bosque mesófilo y de pino-encino-liquidámbar desde el punto de vista de conservación de los mamíferos y también de otros grupos faunísticos (Horváth et al., 2001; Ruíz et al., 1998). Sin embargo, en la actualidad existen escasos lugares en la zona donde persisten estos tipos de vegetación en buen estado de conservación y están limitados en parches pequeños y distantes uno de otro. Los bosques de pino-encino y pino, aunque albergan menor diversidad de mamíferos, son también indispensables para el mantenimiento de la biodiversidad de la región ya que actualmente constituyen la gran mayoría de la superficie boscosa en el PNLM y sus alrededores (March y Flamenco, 1996).

Finalmente, el hecho de que más de la mitad de las especies de mamíferos registradas en este estudio son nuevos para el área a pesar de que es uno de los grupos zoológicos más estudiados y conspicuos, confirma que el nivel del conocimiento 
sobre los recursos bióticos del parque es todavía pobre. Por lo anterior, resaltamos la necesidad de obtener información de manera integral sobre los procesos ecológicos y sociales en el área que servirán como base para el desarrollo de estrategias de conservación viables y adecuadas para rescatar el PNLM.

\section{AGRADECIMIENTOS}

Agradecemos a R. Martínez, R. Castellanos, M. Reyes y J. Bolaños por su colaboración durante el trabajo de campo. Este trabajo se pudo realizar gracias al financiamiento otorgado por el Sistema de Investigación Regional Benito Juárez del CONACyT (SIBEJ-, CHIS-RNA-056-96), Fondo Mexicano para la Conservación de la Naturaleza, A.C. (FMCN, B1-96/325) y el Consejo Nacional para la Ciencia y Tecnología (CONACyT, 323201-5-4189PN). Parte del equipo de campo fue donado por Idea Wild. Finalmente, agradecemos a R. Medellín, G. Csorba, L. Ruíz, así como al ECOSUR, al personal del Parque Nacional Lagunas de Montebello, así también a los habitantes de las comunidades de Antelá, Nueva Rosita, San Rafael El Arco, Santiago El Vértice y Yalmuz-Ojo de Agua, y al grupo de guías de turista de Bosque Azul.

\section{LITERATURA CITADA}

Aranda, M. J. e I. J. March. 1987. Guía de los mamíferos silvestres de Chiapas. Instituto Nacional de Investigaciones sobre Recursos Bióticos, Xalapa,Veracruz.

Arita, H. T.y G. Ceballos. 1997. Los mamíferos de México: distribución y estado de Conservación. Revista Mexicana de Mastozoología, 2:33-71.

Baillie, J. y B. Groombridge. 1996. IUCN red list of threatened animals. IUCN, Gland, Suiza.

Baker, R. H., R. G. Webb y E. Stern. 1971. Amphibians, reptiles and mammals from NorthCentral Chiapas. Anales del Instituto de Biología, Universidad Nacional Autónoma de México. Serie Zoología, 42(1):77-86.

Carlson, M. C. 1954. Floral elements of the pine-oak-liquidambar forest of Montebello, Chiapas, Mexico. Bulletin of Torrey Botanical Club, 81(5):387-399.

Cervantes, F. A., G. Matamoros-Trejo e I. Martínez-Mateos. 1995. Mamíferos silvestres de la unidad de evaluación y monitoreo de la biodiversidad “Ing. Luis Macías Arellano”, San Cayetano, Estado de México. Anales del Instituto de Biología, Universidad Nacional Autónoma de México. Serie Zoología, 66(2):233-239.

CITES. 1995. Appendices I, II, III, to the Convention on International Trade in Endangered Species of Wild Fauna and Flora. CITES Parties at the Ninth Conference of the Parties. U.S. Fish and Wildlife Service, 1-23.

Espinoza, E., A. Anzures y E. Cruz. 1998. Mamíferos de la Reserva de la Biosfera El Triunfo, Chiapas. Revista Mexicana de Mastozoología, 3:79-94.

Flores-Villela, O. y P. Gerez. 1988. Conservación en México: Síntesis sobre vertebrados terrestres, vegetación y uso de suelo. CONABIO-UNAM. México, D. F. 
Hall, E. R. 1981. The Mammals of North America. John Wiley and Sons, New York.

Horváth, A. y D. A. Navarrete-Gutiérrez. 1997. Ampliación del área de distribución de Peromyscus zarhynchus Merriam, 1898 (Rodentia: Muridae). Revista Mexicana de Mastozoología, 2:122-125.

Horváth, A., I. J. March y J. H. D. Wolf. 2001. Rodent diversity and land use in Montebello, Chiapas, México. Studies on Neotropical Fauna and Environment, 36:169-176.

Huckaby, D. G. 1980. Species limits in the Peromyscus mexicanus group (Mammalia: Rodentia: Muroidea). Contribution of Science, Natrural History Museum Los Angeles County, 326:1-24.

March, I. J. y A. Flamenco. 1996. Evaluación rápida de la deforestación en las áreas naturales protegidas de Chiapas (1970-1993). ECOSUR-The Nature Conservancy-U.S.AID. San Cristóbal de Las Casas, Chiapas.

March, I. J., A. Muñoz, D. Navarrete, C. Macías, M. P. Alba, M. Fuller, M. E. Utrera, R. Domínguez, R. M. Vidal, P. Bubb, Y. Reyes e I. Fuentes. 1995. Evaluación y análisis geográfico de la diversidad faunística de Chiapas (Primera Etapa). ECOSURECOSFERA-Pronatura. Informe para la Comisión Nacional para el Uso y Conocimiento de la Biodiversidad. San Cristóbal de Las Casas, Chiapas.

Medellín, R. A., H. T. Arita y O. Sánchez H. 1997. Identificación de los murciélagos de México. Publicaciones Especiales Núm. 2. Asociación Mexicana de Mastozoología, A. C., México D. F.

Medellín, R. A. 1994. Mammal diversity and conservation in the Selva Lacandona, Chiapas, Mexico. Conservation Biology, 8:780-799.

Melo, G. C. y J. Cervantes. 1986. Propuestas para el programa integral de manejo y desarrollo del Parque Nacional Lagunas de Montebello. Instituto de Geografía, Boletín No. 16. Universidad Nacional Autónoma de México, México D. F.

Navarrete, D. A., M. P. Alba, I. J. March y E. Espinoza. 1996. Mamíferos de la Selva El Ocote, Chiapas. Pp. 179-207, en: Conservación y desarrollo sustentable en la Selva El Ocote, Chiapas. (M. A. Vázquez e I. J. March, eds.). ECOSUR-ECOSFERA-CONABIO, San Cristóbal de Las Casas, Chiapas.

Peña-Jiménez, A., L. D. Smith y C. A. Echegaray. 1998. Conservación. Pp. 183-210, en: La diversidad biológica de México. CONABIO, México, D. F.

Reid, F. A. 1997. A field guide to the mammals of Central America y Southeast Mexico. Oxford University Press, New York.

Retana, O. G. y C. Lorenzo. 2001. Lista de los mamíferos terrestres de Chiapas: endemismo y estado de conservación. Acta Zoológica Mexicana (n.s.), 85:25-49.

Reyes, Y. M. P. 2001. Estudio poblacional de Peromyscus zarhynchus (Rodentia: Muridae) en el Parque Nacional Lagos de Montebello, Chiapas, México. Tesis de Licenciatura. Universidad de Guadalajara, Zapopan, Jalisco.

Ruíz, L., A. Horváth, R. Martínez, P. Enríquez-Rocha y J. L. Rangel. 1998. Situación actual de la fauna silvestre en el Parque Nacional Lagos de Montebello: Curso-taller de orientación ecológica (Chis-RNA-056-96). Informe Final. ECOSUR-SIBEJ, San Cristóbal de Las Casas, Chiapas.

Sarmiento A., R. 1999. Estudio poblacional de tres especies de roedores (Rodentia: Muridae) 
en el Parque Nacional Lagos de Montebello, Chiapas. Tesis de Licenciatura. Universidad de Ciencias y Artes del Estado de Chiapas, Tuxtla Gutiérrez, Chiapas.

Secretaria de Agricultura y Ganadería. 1956. Decreto que declara necesaria la utilidad y de utilidad publica la creación de un Parque Nacional en la región conocida con el nombre de Lagunas de Montebello, ubicada en la Independencia y Trinitaria, Chiapas. Diario Oficial de la Federación, 7: 1.0004.

SEMARNAT. en prensa. Norma Oficial Mexicana NOM-059-ECOL-2001, Protección ambiental-Especies nativas de México de flora y fauna silvestres-Categorías de riesgo y especificaciones para su inclusión, exclusión o cambio-Lista de especies en riesgo. Diario Oficial de la Federación, México D. F.

Smith, J. D. \& J. K. Jones, Jr. 1967. Additional records of the Guatemalan Vole, Microtus guatemalensis Merriam. The Southwestern Naturalist, 12:189-191.

Thomas, D. W. y R. K. LaVal. 1988. Survey and census methods. Pp. 77-90, en: Ecological and behavioral methods for the study of bats. (T. H. Kunz, ed.). Smithsonian Institute Press, Washington D. C.

Vidal, R. 1998. Los mamíferos de Coapilla, Chiapas, México. Tesis de Licenciatura. Universidad de Ciencias y Artes del Estado de Chiapas, Tuxtla Gutiérrez, Chiapas.

Wilson, D. E., F. R. Cole, J. D. Nichols, R. Rudran y M. S. Foster (eds.). 1996. Measuring and monitoring biological diversity. Standard methods for Mammals. Smithsonian Institution Press, Washington, D. C.

Zarza, H. 2001. Estructura de la comunidad de pequeños mamíferos en diversos hábitats en la Selva Lacandona, Chiapas. Tesis de Licenciatura, FES Iztacala, UNAM, Iztacala. 

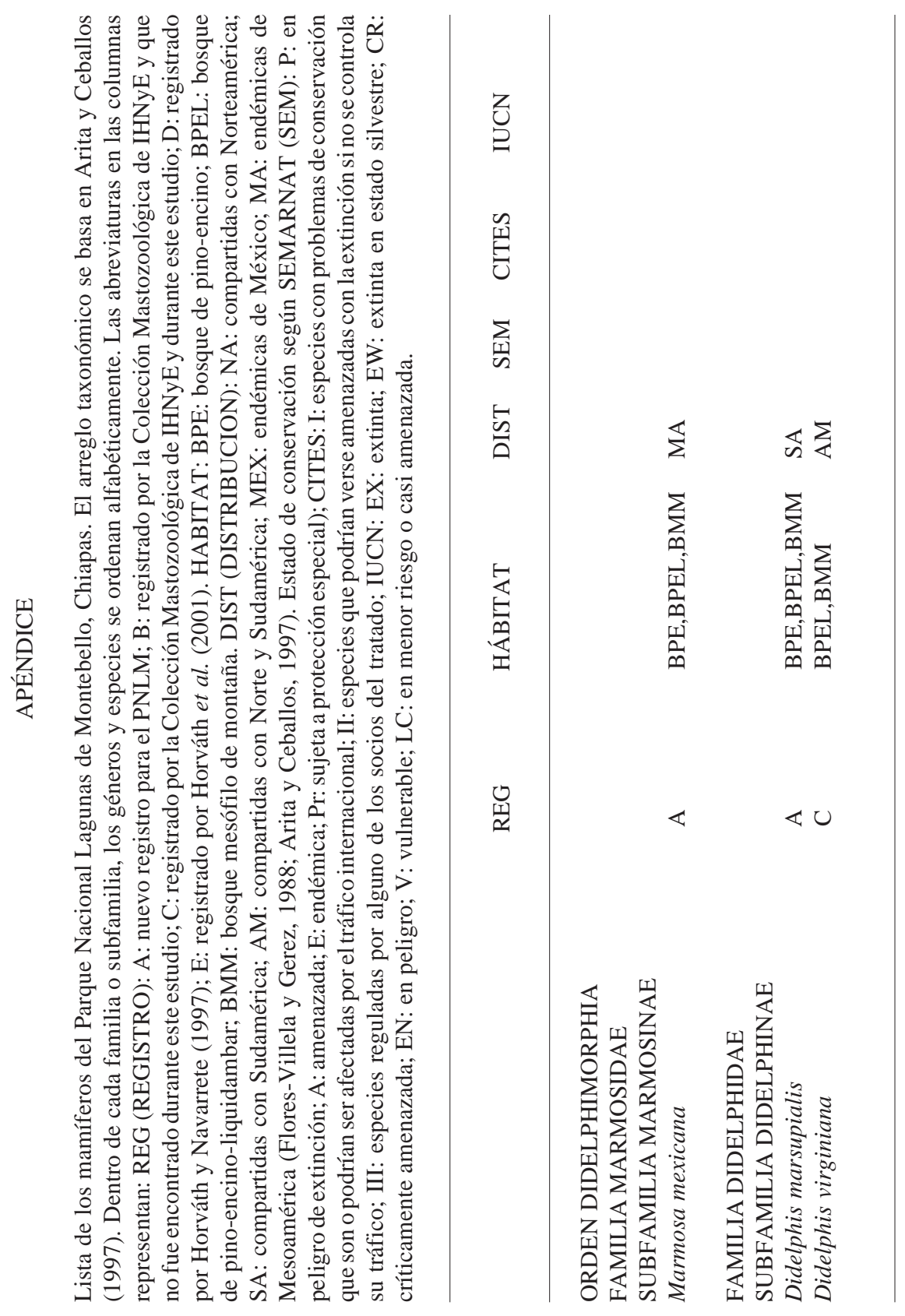


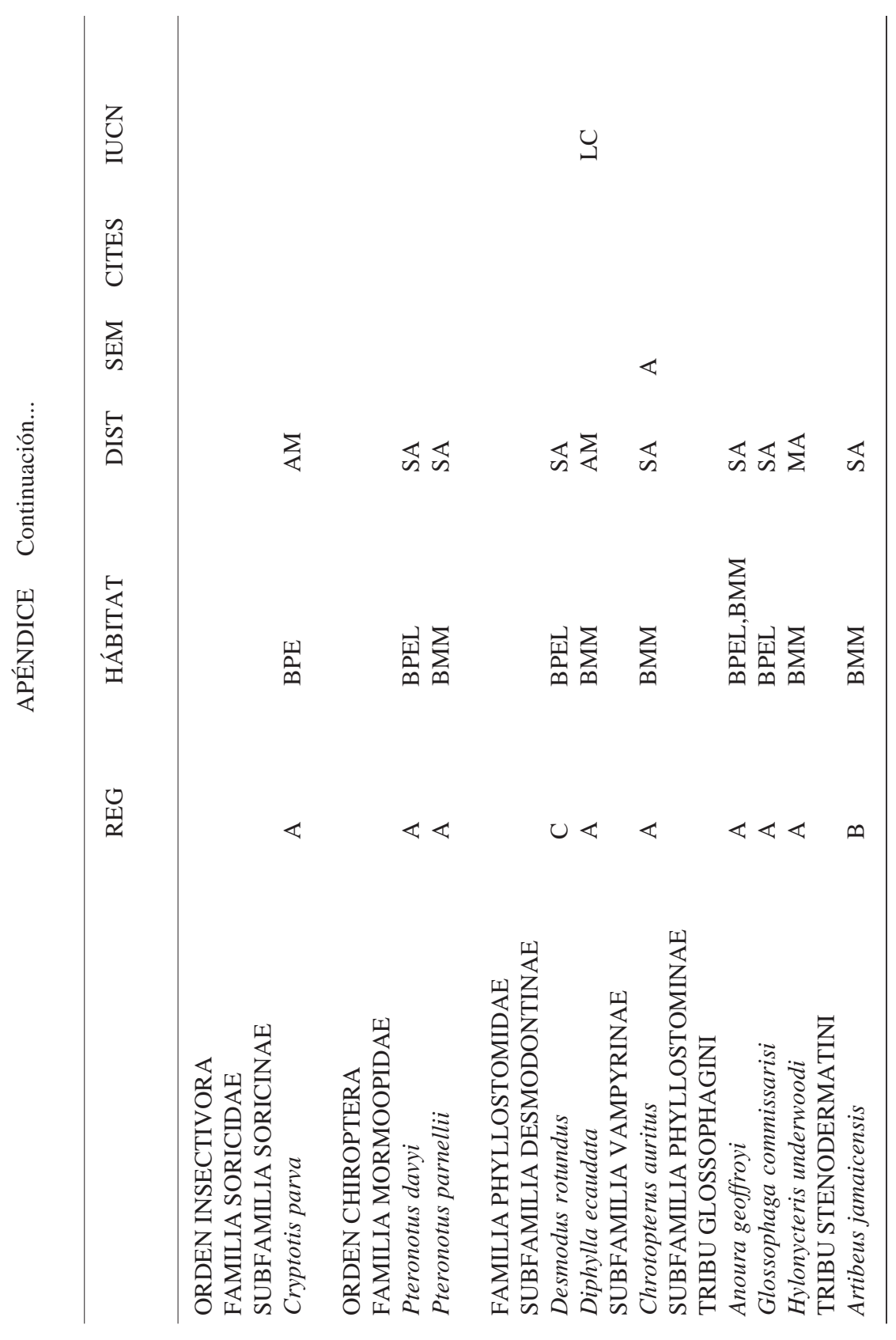




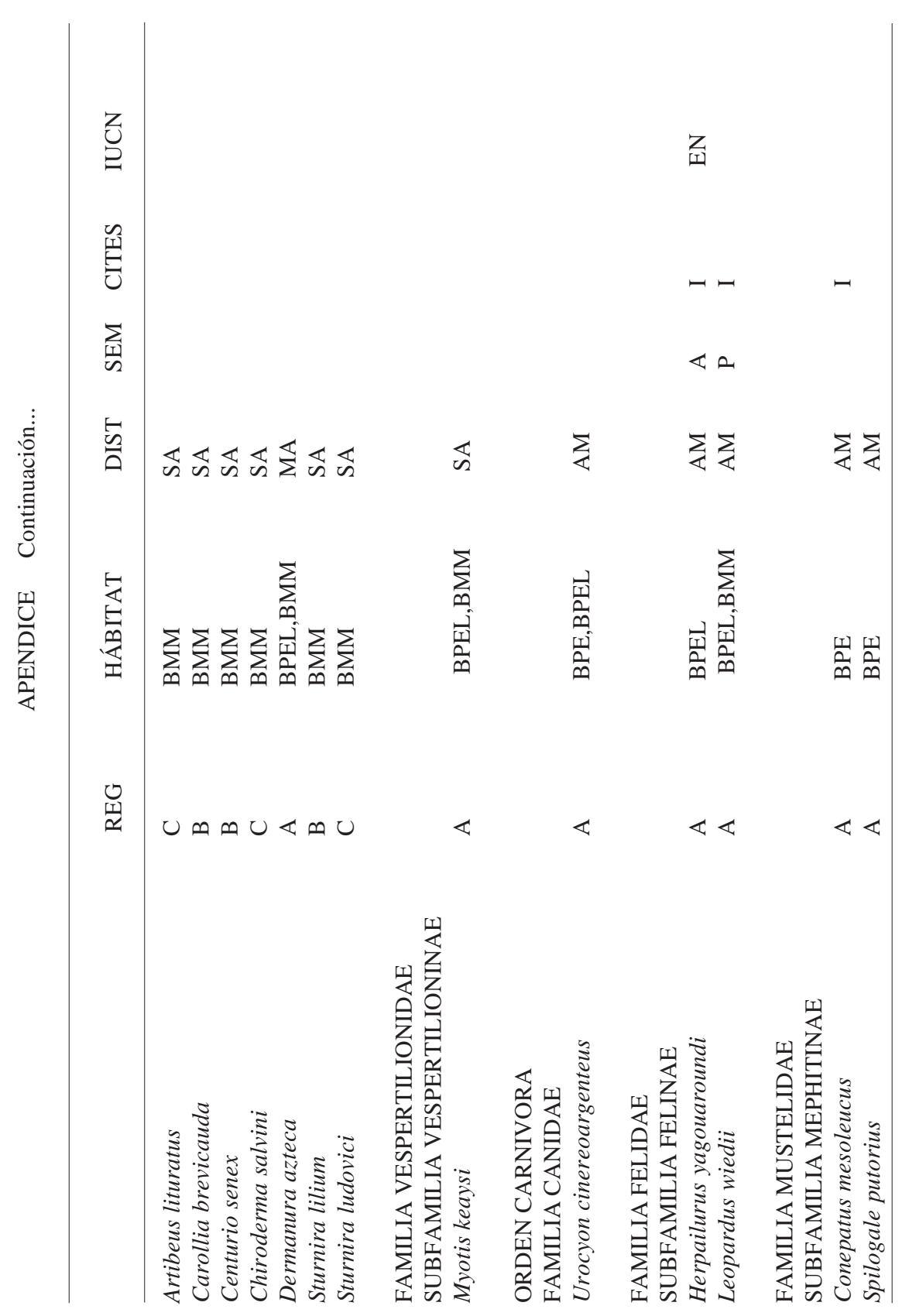




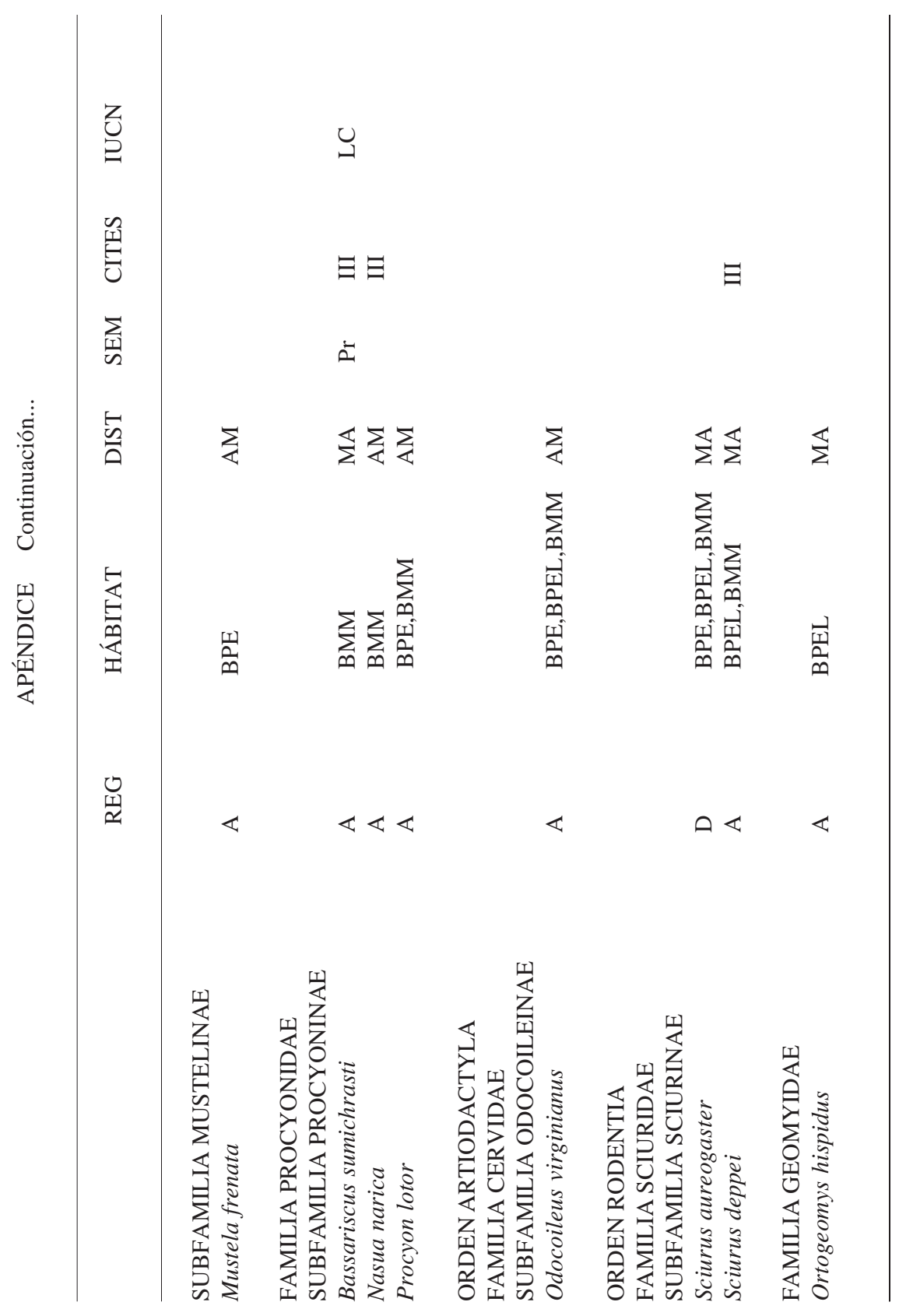




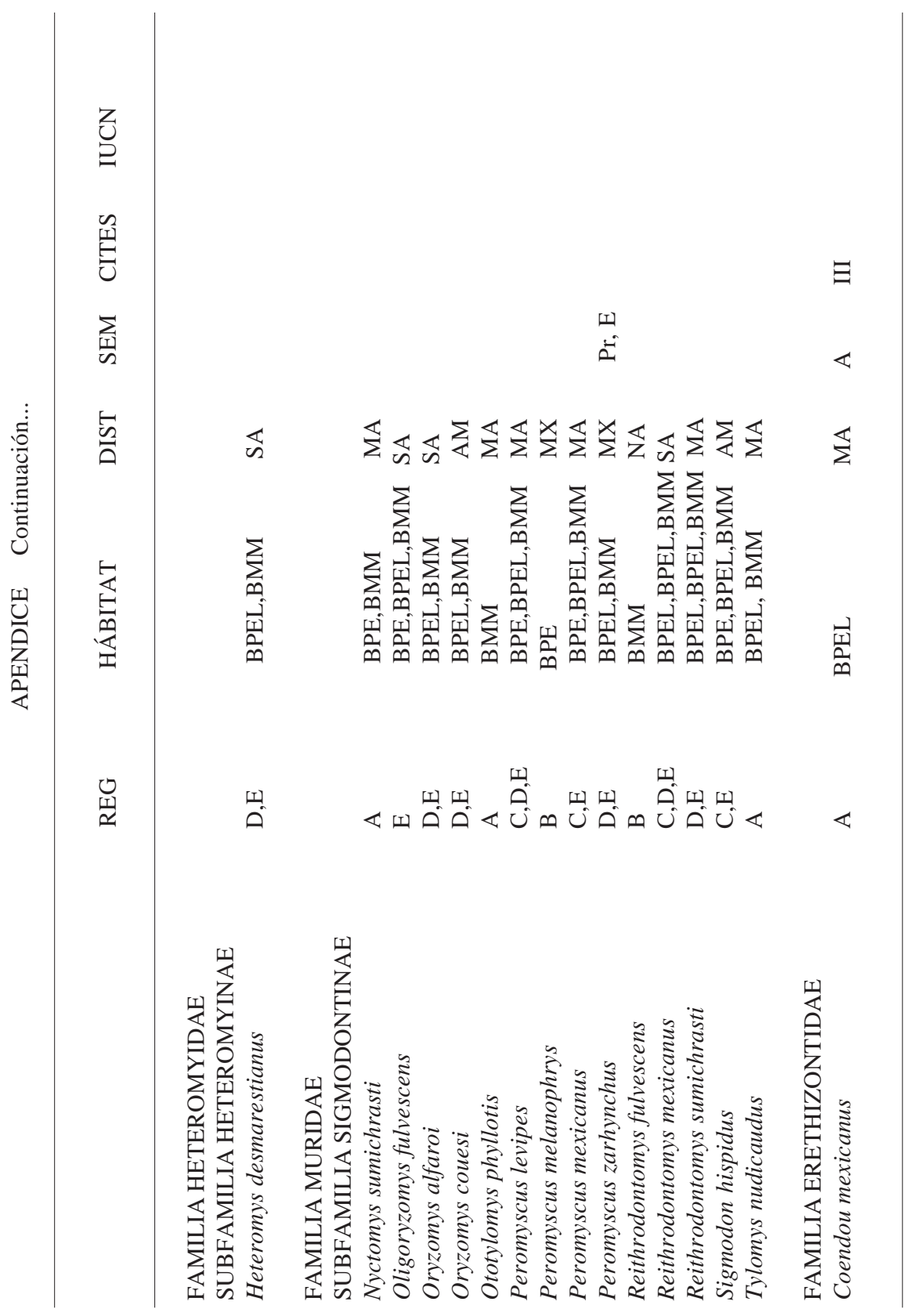


vol. 5

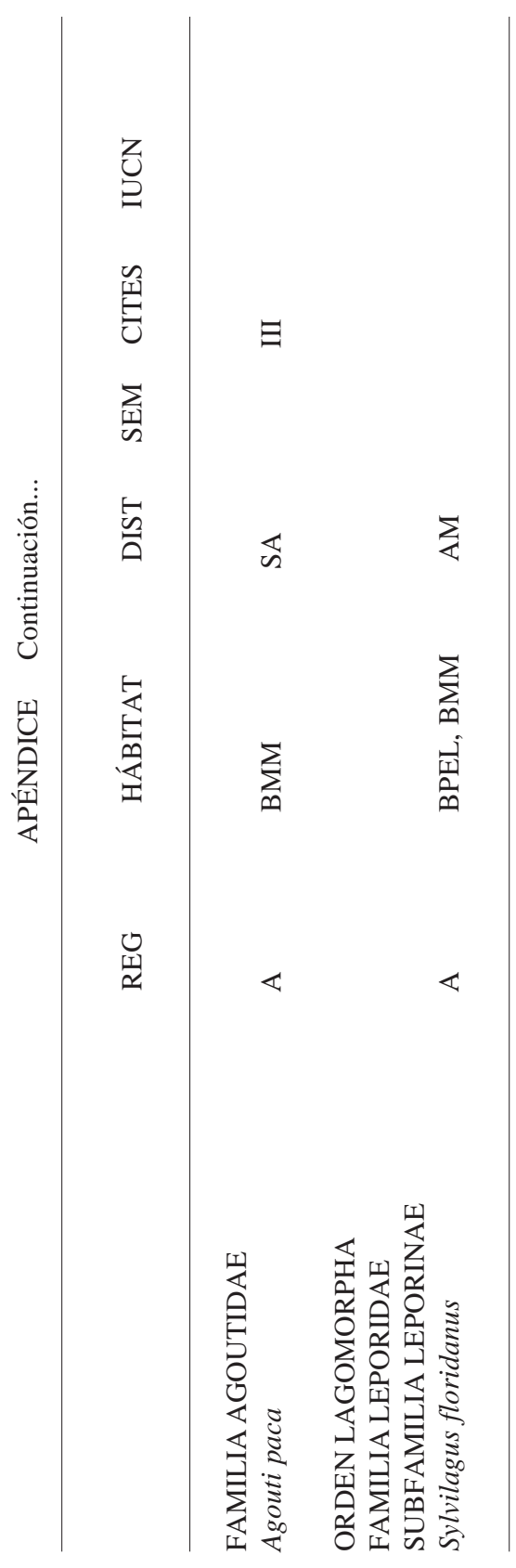

